OPEN ACCESS

Edited by:

Speranta lacob,

Fundeni Clinical Institute, Romania

Reviewed by:

Shinkichi Takamori,

National Hospital Organization Kyushu

Cancer Center, Japan

Anna Mrzljak

University of Zagreb, Croatia

Guoying Wang,

Third Affiliated Hospital of Sun Yat-sen

University, China

*Correspondence:

Gabriele Spoletini

gabriele.spoletini@policlinicogemelli.it

Specialty section:

This article was submitted to Clinical Nutrition,

a section of the journal

Frontiers in Nutrition

Received: 12 October 2021 Accepted: 06 December 2021

Published: 07 January 2022

Citation:

Spoletini G, Ferri F, Mauro A

Mennini G, Bianco G, Cardinale V,

Agnes S, Rossi M, Avolio AW and

Lai Q (2022) CONUT Score Predicts

Early Morbidity After Liver

Transplantation: A Collaborative Study.

Front. Nutr. 8:793885

doi: 10.3389/fnut.2021.793885

\section{CONUT Score Predicts Early Morbidity After Liver Transplantation: A Collaborative Study}

\author{
Gabriele Spoletini ${ }^{*}$, Flaminia Ferri ${ }^{2}$, Alberto Mauro ${ }^{1}$, Gianluca Mennini ${ }^{2}$, \\ Giuseppe Bianco ${ }^{1}$, Vincenzo Cardinale ${ }^{2}$, Salvatore Agnes ${ }^{1}$, Massimo Rossi ${ }^{2}$, \\ Alfonso Wolfango Avolio ${ }^{1}$ and Quirino Lai $^{2}$ \\ ' General Surgery and Liver Transplantation, Fondazione Policlinico Universitario Agostino Gemelli IRCCS, Rome, Italy, \\ ${ }^{2}$ General Surgery and Organ Transplantation Unit, Sapienza University of Rome, Rome, Italy
}

Introduction: Liver transplantation $(\mathrm{LT})$ is burdened by the risk of post-operative morbidity. Identifying patients at higher risk of developing complications can help allocate resources in the perioperative phase. Controlling Nutritional Status (CONUT) score, based on lymphocyte count, serum albumin, and cholesterol levels, has been applied to various surgical specialties, proving reliable in predicting complications and prognosis. Our study aims to investigate the role of the CONUT score in predicting the development of early complications (within 90 days) after LT.

Methods: This is a retrospective analysis of 209 patients with a calculable CONUT score within 2 months before LT. The ability of the CONUT score to predict severe complications, defined as a Comprehensive Complication Index $(\mathrm{CCI}) \geq 42.1$, was examined. Inverse Probability Treatment Weighting was used to balance the study population against potential confounders.

Results: Patients with a $\mathrm{CCl} \geq 42.1$ had higher CONUT score values (median: 7 vs. 5 , $P$-value $<0.0001)$. The CONUT score showed a good diagnostic ability regarding post-LT morbidity, with an AUC $=0.72(95.0 \% \mathrm{Cl}=0.64-0.79$; $P$-value $<0.0001)$. The CONUT score was the only independent risk factor identified for a complicated post-LT course, with an odds ratio $=1.39(P$-value $<0.0001)$. The 90 -day survival rate was $98.8 \%$ and $87.5 \%$ for patients with a CONUT score $<8$ and $\geq 8$, respectively.

Conclusions: Pre-operative CONUT score is a helpful tool to identify patients at increased post-LT morbidity risk. Further refinements in the score composition, specific to the LT population, could be obtained with prospective studies.

Keywords: nutrition, immunology, post-operative morbidity, liver transplant complications, cholesterol, albumin, lymphocyte count

\section{INTRODUCTION}

Liver transplantation (LT) is the cure for a growing number of patients with end-stage liver disease. Many patients who were once deemed too frail are now considered for LT (1). However, due to the necessity to fulfill the gap between offer and demand of liver grafts, increased utilization of extended-criteria donors has led to more risky donor-to-recipient matches (2). These challenging matches contribute to post-operative morbidity and poor long-term outcomes (3). 
With the intent to identify frail patients with a greater post-LT risk of complications, sophisticated scores have been introduced focusing on graft function recovery and efficacious retransplantation $(4,5)$. Malnutrition and immunological status can influence treatment outcomes, with various studies weighing their impact after surgery (6-8). The Controlling Nutritional Status (CONUT) score has been developed to measure both aspects and has been trialed in different settings, including cancer surgery and oncologic treatments (9-12). The CONUT score has been tested with the intent to predict overall survival and hepatocellular cancer (HCC) recurrence after LT and post-operative complications in pancreatic, esophageal, gastrointestinal, and orthopedic surgery (13-16).

However, the ability of the CONUT score to predict postLT early morbidity and mortality has not been investigated yet. The primary aim of the study was to investigate the role of the CONUT score calculated before LT in predicting the development of severe post-transplant complications as graded by the Comprehensive Complication Index (CCI). The secondary aim was to investigate the role of the pre-LT CONUT score in predicting post-operative mortality within 90 days post-LT.

\section{MATERIALS AND METHODS}

\section{Study Design}

This is a retrospective bicentric observational study investigating the data of patients undergoing LT.

The Strengthening the Reporting of Observational Studies in Epidemiology (STROBE) guidelines were followed to create the study.

\section{Setting}

The participant centers were Sapienza University of Rome, Umberto I Polyclinic of Rome, and Catholic Rome University, Gemelli Hospital.

\section{Population}

A total of 209 cases transplanted at Sapienza Rome University (period January 2013-December 2020) and Catholic Rome University (period September 2016-December 2020) were considered for the analysis. The only inclusion criterion was the availability of enough data for calculating the CONUT, and the CCI scores were enrolled for the study.

All the study subjects were adult ( $\geq 18$ years) patients receiving a graft from a deceased-brain donor, including split grafts and retransplants.

\section{Outcomes}

The primary outcome of the study was the development of a complex post-operative course defined as a CCI $\geq 42.1$. The secondary outcome was the post-LT 90-day mortality. The last follow-up date was May 31st, 2021.

\section{Data Collection}

Data were retrospectively obtained from the prospectively collected charts of the patients. The guarantor of the data quality was the Data Manager of the Study Group (QL). Data errors and missingness were identified across the database and solved, when possible, with specific queries.

\section{Definitions}

The CONUT score was calculated according to the original descriptions (9-12). The CONUT score is based on serum albumin, cholesterol, and total lymphocyte count. (12) CONUT score ranges from 0 (i.e., normal nutritional status) to 12 (i.e., severe malnutrition) (Table 1). The CONUT score was calculated using the last available data from blood tests of patients on the LT waitlist. We arbitrarily decided to select an upper limit of 2 months before LT for calculating the score: all the patients with data older than 2 months before the transplant were excluded from the study.

The CCI is a recently proposed classification for evaluating post-operative complications. This score is more sophisticated respect to the more commonly used Dindo-Clavien classification system (17). The CCI carries the advantage of capturing the burden of the entire morbidity rather than grading only the most severe complication (18). Dindo-Clavien grade I corresponds to 8.7, grade II to 20.9, grade IIIa to 26.2, grade IIIb to 33.7, grade IVa to 42.4 , grade IVb to 46.2 , and grade V to 100 . In the liver transplantation setting, CCI has shown a good prediction ability for 90-day and 1-year graft loss risk (19). The CCI ranges from 0 (i.e., absence of post-operative complications) to 100 (i.e., death) $(12,18)$. A web-calculator was used for estimating CCI (available at https://www.assessurgery.com). The CCI was calculated using the following original algorithm: CCI $=\left[\sqrt{ }\left(w_{\mathrm{wC}}+{ }_{\mathrm{wC}} \ldots\right.\right.$ $+\mathrm{wCx})] / 2$.

All the complications collected were summed, even if the same patient received several times multiple administrations of the same medical (i.e., blood transfusion) or interventional (i.e., various radiological or surgical approaches) treatment. In the present study, the entire population was categorized into two groups according to the presence of a low $(<42.1)$ or high $(\geq 42.1)$ CCI value. The CCI threshold value of 42.1 was set according to previously published studies (20). The CCI value was calculated at the time of discharge after LT.

\section{Statistical Analysis}

Continuous variables were reported as medians and interquartile ranges (IQR). Categorical variables were reported as numbers and percentages. Mann-Whitney $U$ test and Fisher's exact test were used to compare continuous and categorical variables, respectively.

Missing data relative to study covariates always involved $<10 \%$ of patients. In all the cases, missing data were handled with a single imputation method. In detail, a median of nearbypoints imputation was adopted. The median instead of the mean was adopted due to the skewed distribution of the managed variables (21).

With the intent to compensate for the non-randomized design of this retrospective study, the population was "balanced" using Inverse Probability Treatment Weighting (IPTW). With the intent to perform the comparison between low and high CCI groups, twelve potential confounders were included in the model: patient age, patient male sex, HCC, hepatitis C virus (HCV) 
TABLE 1 | Controlling nutritional status score calculation.

\begin{tabular}{lcccc}
\hline Variables & \multicolumn{4}{c}{ Undernutrition status } \\
\cline { 2 - 5 } & Normal & Light & Moderate & Severe \\
\hline Albumin (g/dL) & $\geq 3.5$ & $3.0-3.49$ & $2.5-2.9$ & $<2.5$ \\
Points & 0 & 2 & 4 & 6 \\
Total lymphocyte count $\left(\mathbf{m m}^{\mathbf{3}}\right)$ & $>1,600$ & $1,200-1,599$ & $800-1,199$ & $<800$ \\
Points & 0 & 1 & 2 & 3 \\
Total cholesterol (mg/dL) & $>180$ & $140-180$ & $100-139$ & $<100$ \\
Points & 0 & 1 & 2 & 3 \\
Total CONUT score & $0-1$ & $2-4$ & $5-8$ & $9-12$ \\
\hline
\end{tabular}

positive status, acute liver failure, waiting list duration, MELDNa, donor age, donor male sex, cold ischemia time (CIT), piggyback caval reconstruction, cava replacement with veno-venous bypass (VVB).

With the intent to reduce the artificial increase of the sample size, and, therefore, of the type I error rate (namely, the increased number of false positives) caused by the inflated sample size in the pseudo data, we used stabilized weights (SW) according to the formula:

$$
\begin{aligned}
& \mathrm{SW}=\mathrm{p} / \mathrm{PS} \text { for the study group; } \\
& \mathrm{SW}=(1-\mathrm{p}) /(1-\mathrm{PS}) \text { for the control group }
\end{aligned}
$$

where $p$ is the probability of etiology without considering covariates and PS is the propensity score.

Because $p$-values can be biased from population size, results from the comparisons between covariates subgroups were reported as effect size ( $D$ value): values $<|0.1|$ indicated very small differences between means, values between $|0.1|$ and $|0.3|$ indicated small differences, values between $|0.3|$ and $|0.5|$ indicated moderate differences, and values $>|0.5|$ indicated considerable differences (22).

A multivariable logistic regression model was developed in the post-IPTW population for the risk of $\mathrm{CCI} \geq 42.1$. Odds ratios (ORs) and 95\% confidence intervals $(95 \% \mathrm{CI}$ ) were reported. A backward conditional method was used for identifying the risk factors for high CCI.

The accuracy of the CONUT score was assessed for the risk of CCI $\geq 42.1$ through the Harrel's c statistic. The area under the curve (AUC) and 95\% CIs were reported. The model accuracy was compared with five other variables: MELDNa, MELD, D-MELD, waiting time duration, and CIT. Separate AUC of ROC curves were calculated and analyzed for comparing the single components of the CONUT score (albumin, lymphocyte, and cholesterol).

Ninety-day patient death rates were evaluated using the Kaplan-Meier method, and the log-rank test was adopted to compare the obtained survivals.

Variables with a $P<0.05$ were considered statistically significant. We used the SPSS statistical package version 27.0 (SPSS Inc, Chicago, IL, USA) for the statistical analyses.
TABLE 2 | Comparison between the Low- $(<42.1)$ and the High-CCI $(\geq 42.1)$

\begin{tabular}{|c|c|c|c|}
\hline \multirow[t]{2}{*}{ Variables } & $\begin{array}{c}\mathrm{CCl}<42.1 \\
(n=151)\end{array}$ & $\begin{array}{c}\mathrm{CCl} \geq 42.1 \\
(n=58)\end{array}$ & \multirow[t]{2}{*}{$P$} \\
\hline & \multicolumn{2}{|c|}{ Median (IQR) or $\mathrm{n}(\%)$} & \\
\hline \multicolumn{4}{|l|}{ Recipient } \\
\hline Age, years & $58(51-63)$ & $57(47-63)$ & 0.26 \\
\hline Male sex & $128(84.8)$ & $54(93.1)$ & 0.17 \\
\hline Height, cm & $170(165-177)$ & $175(169-177)$ & 0.13 \\
\hline Weight, kg & $76(65-87)$ & $80(68-89)$ & 0.52 \\
\hline $\mathrm{BMI}$ & 26 (23-29) & $26(23-29)$ & 0.75 \\
\hline Waiting time duration, months & $4(1-10)$ & $3(0-7)$ & 0.10 \\
\hline $\mathrm{HCC}$ & 85 (56.3) & $22(37.9)$ & 0.02 \\
\hline \multicolumn{4}{|l|}{ Underlying liver disease* } \\
\hline $\mathrm{HCV}$ & 50 (33.1) & $11(19.0)$ & 0.06 \\
\hline HBV & $29(19.2)$ & $10(17.2)$ & 0.84 \\
\hline Alcohol & 59 (39.1) & $21(36.2)$ & 0.75 \\
\hline $\mathrm{NASH}$ & $32(21.2)$ & $15(25.9)$ & 0.47 \\
\hline Biliary cirrhosis & $7(4.6)$ & $4(6.9)$ & 0.50 \\
\hline ALF & $4(2.6)$ & 5 (8.6) & 0.12 \\
\hline Other & $20(13.2)$ & $7(12.1)$ & 1.00 \\
\hline T2DM & $42(27.8)$ & $18(31.0)$ & 0.73 \\
\hline Requiring insulin & $26(17.2)$ & $11(19.0)$ & 0.84 \\
\hline Arterial hypertension & $32(21.2)$ & $9(15.5)$ & 0.44 \\
\hline CONUT & $5(3-7)$ & $7(5-9)$ & $<0.0001$ \\
\hline Albumin (g/L) & $36(31-40)$ & $31(26-35)$ & $<0.0001$ \\
\hline Total cholesterol (mg/dL) & $129(91-159)$ & $100(71-137)$ & 0.001 \\
\hline Lymphocyte count ${ }^{\star} 10^{9} / \mathrm{L}$ & $1.03(0.71-1.41)$ & $0.86(0.62-1.29)$ & 0.09 \\
\hline MELD & $16(10-23)$ & $21(15-30)$ & 0.001 \\
\hline MELDNa & $18(11-25)$ & $23(17-29)$ & 0.001 \\
\hline D-MELD & $853(517-1,288)$ & $1,128(723-1,601)$ & 0.002 \\
\hline \multicolumn{4}{|l|}{ Donor } \\
\hline Age, years & $58(45-71)$ & $63(46-74)$ & 0.42 \\
\hline Male sex & $77(51.0)$ & $23(39.7)$ & 0.17 \\
\hline Height, cm & $167(160-175)$ & $165(160-171)$ & 0.30 \\
\hline Weight, kg & $72(65-85)$ & $71(62-78)$ & 0.37 \\
\hline $\mathrm{BMl}$ & $26(23-28)$ & $26(24-28)$ & 0.95 \\
\hline \multicolumn{4}{|l|}{ Cause of death } \\
\hline CVA & $105(69.5)$ & $39(67.2)$ & 0.74 \\
\hline Blunt trauma & $36(23.8)$ & $18(31.0)$ & 0.30 \\
\hline Anoxia & $8(5.3)$ & $1(1.7)$ & 0.45 \\
\hline Other & $2(1.3)$ & $0(-)$ & 1.00 \\
\hline T2DM & 17 (11.3) & $9(15.5)$ & 0.48 \\
\hline Requiring insulin & $5(3.3)$ & $2(3.4)$ & 1.00 \\
\hline Arterial hypertension & $64(42.4)$ & $26(44.8)$ & 0.76 \\
\hline \multicolumn{4}{|l|}{ Transplant } \\
\hline CIT, minutes & $420(370-450)$ & $450(420-518)$ & $<0.0001$ \\
\hline Piggy-back caval reconstruction & $116(76.8)$ & $29(50.0)$ & $<0.0001$ \\
\hline Temporary portocaval shunt & $28(18.5)$ & $13(22.4)$ & 0.56 \\
\hline Cava replacement with WB & $6(4.0)$ & $14(24.1)$ & $<0.0001$ \\
\hline
\end{tabular}
Group.

*In some cases, more liver diseases were present contemporaneously.

$\mathrm{CCl}$, comprehensive complication index; IQR, interquartile ranges; $n$, number; BMI, body mass index; $H C C$, hepatocellular cancer; $H C V$, hepatitis $C$ virus; $H B V$, hepatitis $B$ virus; NASH, non-alcoholic steato-hepatitis; ALF, acute liver failure; T2DM, type 2 diabetes mellitus; CONUT, Controlling Nutritional Status; MELD, model for end-stage liver disease; Na, sodium; D-MELD, donor-MELD; CVA, cerebrovascular accident; CIT, cold ischemia time; WB, veno-venous bypass. 


\section{RESULTS}

Two hundred and nine patients were included in the study population. The median follow-up period after LT was 37 months (IQR = 17-57). During the follow-up, 32/209 (15.3\%) patients died, of whom $13(6.2 \%)$ within 90 days from LT. In all the early deaths, the cause was a liver-specific condition (i.e., technical problems in six patients and graft failure in seven). In the late deaths, 13 patients died for liver-specific conditions (biliary complications, vascular complications, liver disease recurrence, acute rejection), while six died due to nonliver-specific conditions.

Patient characteristics are reported in Table 2. Several differences were reported between the two groups. Patients with a high-CCI value less commonly had HCC (37.9 vs. $56.3 \% ; P=$ 0.02 ) and presented a median higher MELD value (21 vs. $16 ; P$ $<0.0001)$.

In all the cases, the median values of the CONUT score variables were lower in the high-CCI patients. In detail, median albumin value was 31 vs. $36 \mathrm{~g} / \mathrm{L}(P<0.0001)$, median total cholesterol was 100 vs. $129 \mathrm{mg} / \mathrm{dL}(P=0.001)$, and median lymphocyte count was 0.86 vs. $1.03^{*} 10^{9} / \mathrm{L}(P=0.09)$. Consequently, the CONUT score value was significantly superior in the high-CCI group (median: 7 vs. 5; $P<0.0001$ ) (Figure 1).

No statistical differences were observed concerning the donor characteristics. As for the transplant surgical procedure, the CIT was longer in the high-CCI group (450 vs. $420 \mathrm{~min} ; P<0.0001$ ). Piggy-back caval anastomosis was observed less commonly in the high-CCI group (50.0 vs. $76.8 \%$; $P<0.0001$ ), with higher usage of cava replacement with veno-venous bypass (24.1 vs. 4.0; $P<0.0001)$.

A linear correlation was reported between the CCI and the CONUT values, suggesting a potential connection between these two variables. In detail, a statistical significance was observed ( $P$ $<0.0001)$, although the adjusted $\mathrm{R}$ squared value showed low values (10.5\%) (Figure 2).

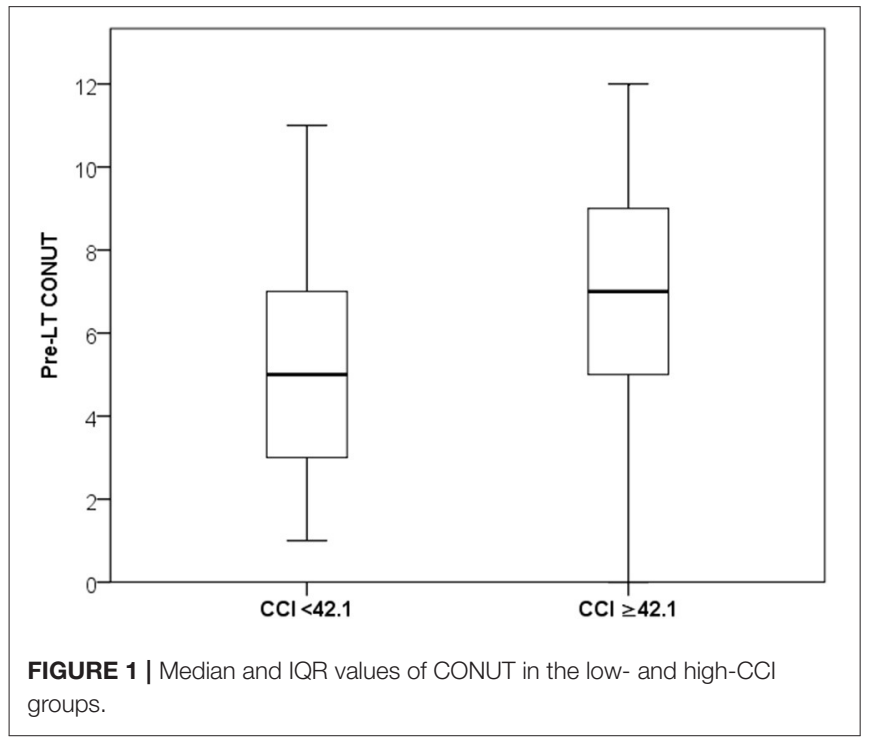

To eliminate potential confounders, the two groups were "balanced" for twelve variables emerging as significantly different between the groups. A stabilized IPTW allowed to reduce the initial differences. As reported in Table 3, also variables initially showing relevant differences of the means such as MELDNa ( $D$-value 0.51), CIT ( $D$-value 0.63), and piggy-back caval reconstruction $(D$-value -0.55$)$, all showed small or very small differences after the IPTW. Thanks to the use of a stabilized approach, the sample size of the pseudo population did not significantly differ with respect to the initial unbalanced population ( 212 vs. 209 cases).

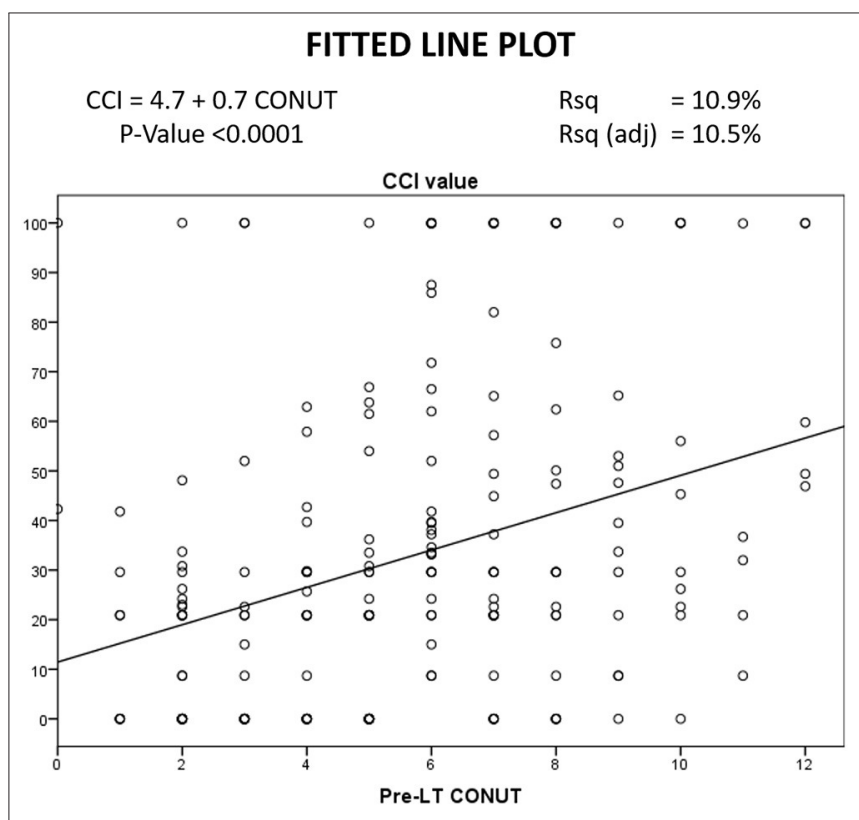

FIGURE 2 | Linear correlation between CONUT and CCI.

TABLE 3 | Effect of IPTW on the variables used for balancing the two groups.

\begin{tabular}{lrc}
\hline & \multicolumn{2}{c}{ Cohen's $D$-value } \\
\cline { 2 - 3 } & Pre-IPTW & Post-IPTW \\
\hline Recipient age & -0.19 & -0.07 \\
Recipient male sex & 0.29 & -0.19 \\
Waiting time duration, months & -0.12 & 0.06 \\
HCC & -0.37 & -0.10 \\
HCV & -0.34 & -0.02 \\
ALF & 0.23 & -0.02 \\
MELDNa & 0.51 & 0.13 \\
Donor age & 0.09 & -0.08 \\
Donor male sex & -0.23 & -0.24 \\
CIT & 0.63 & -0.15 \\
Piggy-back caval reconstruction & -0.55 & -0.05 \\
Cava replacement with WB & 0.09 & -0.02 \\
\hline
\end{tabular}

IPTW, inverse probability therapy weighting; HCC, hepatocellular cancer; HCV, hepatitis $C$ virus; ALF, acute liver failure; MELDNa, model for end-stage liver disease sodium; CIT, cold ischemia time; WB, veno-venous bypass. 
The risk factors for a CCI $\geq 42.1$ were investigated in the postIPTW population using multivariable logistic regression. Twelve different potential risk factors were initially introduced in the mathematical model. Using a backward Wald method, only the CONUT score before LT was an independent risk factor for high CCI. In detail, CONUT showed an OR $=1.39$ (95\%CI $=1.21-$ 1.58; $P<0.0001)$. In other terms, each increase of one point of CONUT score increased the risk of high CCI by 39\% (Table 4). All the other patient-, donor-, and transplant-related variables failed to have a relevant role as risk factors for high CCI.

At c-statistics analysis in the pseudo population, the CONUT score was the unique tested variable showing diagnostic ability, with an $\mathrm{AUC}=0.72(95 \% \mathrm{CI}=0.64-0.79 ; P<0.0001)$. All the other potential diagnostic tools measured at the time of LT (i.e., MELD, MELDNa, D-MELD) failed to predict a high CCI, as reported in Table 5 and Figure 3. All of the single variables of the CONUT score showed significant AUC in terms of prognostic ability for the risk of CCI $\geq 42$, in particular albumin AUC was superimposable to CONUT score AUC (data not shown).

When the post-IPTW population was split according to the CONUT value, the 90-day, 1-year, and 5-year patient death rates were $1.2,4.2$, and $9.1 \%$, respectively, when the CONUT value was $<8$. On the opposite, when a CONUT score $\geq 8$ was observed, the 90-day, 1-year, and 5-year patient death rates increased to 12.5, 14.3 , and $27.0 \%$, with a statistically significant difference between the two subgroups (log-rank $P=0.02$ ) (Figure 4A).

Similar results were observed when only the liver-specific death rates were reported (Figure 4B). In detail, the 90-day, 1year, and 5-year liver-specific death rates were 1.2, 4.2, and 6.6\%, respectively, with CONUT scores $<8$, whilst they increased to $12.5,14.3$, and $20.4 \%$ respectively with CONUT scores $\geq 8$. Also in this case, a statistically significant difference between the two subgroups was reported (log-rank $P=0.09$ ).

\section{DISCUSSION}

Malnutrition and immunologic compromise increase the risk of post-LT complications, particularly after "extended-criteria donor to frail recipient" matches $(23,24)$. The possibility to preoperatively predict this potential risk is pivotal for optimizing resource allocation and preserving LT outcomes.

Our study demonstrated the efficacy of the CONUT score in predicting severe post-LT complications, with an $\mathrm{OR}=1.39$. Moreover, patients with high $(\geq 8)$ pre-transplant CONUT values showed poor post-operative 90-day as well as long-term patient survival rates. In particular, we encountered a higher rate of liverspecific deaths at all the time points analyzed, highlighting the role of the pre-transplant condition of the recipient.

Our findings align with previous studies exploring the predictive role of the CONUT score in the setting of hepatic $(10,25-27)$, thoracic, urological, and gastrointestinal oncological surgery (28-32). A study from China $(N=94)$ showed that pre-operative CONUT was the best predictor of overall and recurrence-free survivals in patients resected for hilar cholangiocarcinoma (10).

A multicenter study from Japan $(N=2461)$ similarly showed that the pre-operative CONUT score was predictive of worse overall and recurrence-free survivals in patients resected for HCC, even after propensity score matching analysis (27).

A study from Japan $(N=204)$ suggested that the CONUT score was a strong independent predictor of survival among stage II/III colorectal cancer patients (28).

As for the setting of LT, only a limited number of studies have been published $(6,33)$.

A study from Italy $(N=280)$ explored the specific impact of CONUT in the LT population with HCC. Of relevance, this study failed to observe any correlation between the CONUT score and post-LT poor survival or tumor recurrence (6). A potential explanation for these results could derive from the super-selection of the explored population. In fact, in LT, HCC patients represent a well-known selected population with a more compensated liver condition and, therefore, a predictable narrower spread of CONUT values.

Another study from the same authors $(N=324)$ investigated the post-LT trend of CONUT in HCC patients, reporting worse values in the early post-LT period than the pre-LT values and a substantial improvement after the post-LT third month (33).

TABLE 4 | Multivariable logistic regression model for the risk of $\mathrm{CCl} \geq 42.1$.

\begin{tabular}{|c|c|c|c|c|c|c|c|}
\hline Variable & Beta & SE & Wald & OR & \multicolumn{2}{|c|}{$95 \% \mathrm{Cl}$} & $\mathbf{P}$ \\
\hline Donor male sex & -0.54 & 0.34 & 2.42 & 0.59 & 0.30 & 1.15 & 0.12 \\
\hline WT duration in months & 0.03 & 0.02 & 2.31 & 1.03 & 0.99 & 1.07 & 0.13 \\
\hline Donor age & -0.004 & 0.01 & 0.22 & 0.996 & 0.98 & 1.01 & 0.64 \\
\hline Recipient male sex & -0.06 & 0.44 & 0.02 & 0.94 & 0.40 & 2.21 & 0.89 \\
\hline Constant & -2.89 & 1.24 & 5.43 & 0.06 & - & - & 0.02 \\
\hline
\end{tabular}

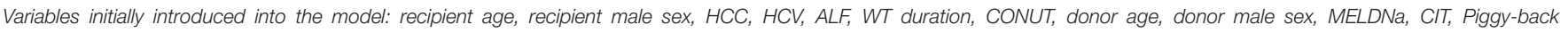
caval reconstruction.

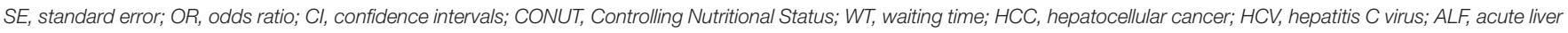
failure; MELDNa, model for end-stage liver disease sodium; CIT, cold ischemia time. 
TABLE 5 | C-statistics for the evaluation of CONUT performance for the diagnosis of $\mathrm{CCl} \geq 42.1$.

\begin{tabular}{llllll}
\hline Variables & AUC & SE & \multicolumn{2}{c}{$95 \% \mathbf{C l}$} & P \\
\cline { 4 - 5 } & & & Lower & Upper & \\
\hline CONUT & 0.72 & 0.04 & 0.64 & 0.79 & $<0.0001$ \\
MELD & 0.58 & 0.04 & 0.50 & 0.66 & 0.06 \\
MELDNa & 0.57 & 0.04 & 0.49 & 0.65 & 0.09 \\
D-MELD & 0.53 & 0.04 & 0.45 & 0.62 & 0.44 \\
WT duration & 0.53 & 0.04 & 0.44 & 0.61 & 0.51 \\
CIT & 0.48 & 0.04 & 0.39 & 0.57 & 0.65 \\
\hline
\end{tabular}

AUC, area under the curve; SE, standard error; Cl, confidence intervals; CONUT, Controlling Nutritional Status; MELD, model for end-stage liver disease; Na, sodium; D-MELD, donor-MELD; WT, waiting time; CIT, cold ischemia time.

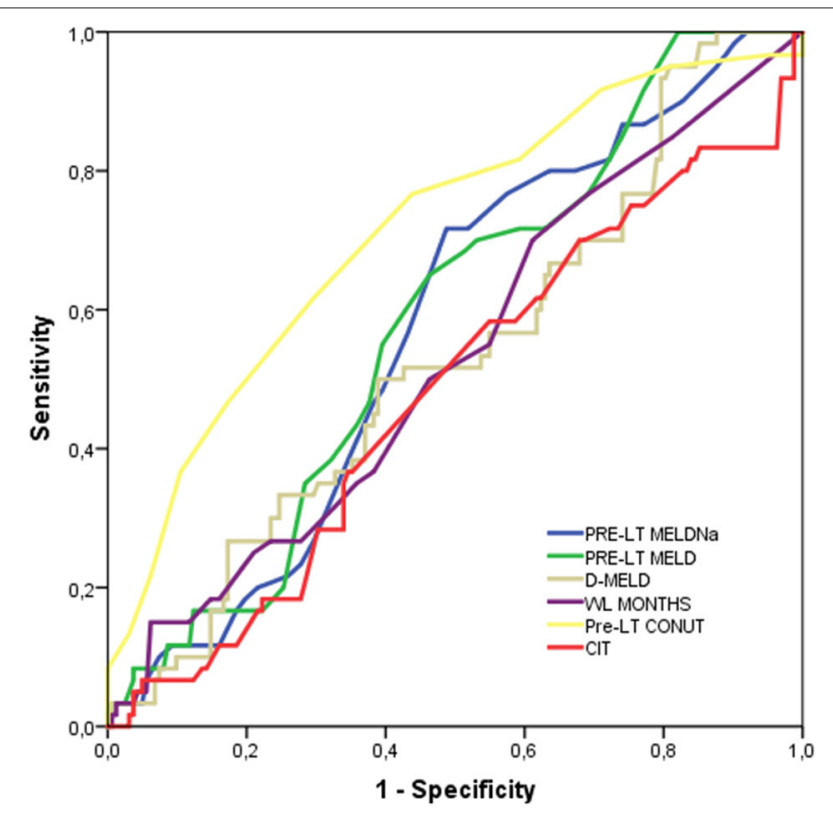

FIGURE 3 | Harrel's c-statistics for the diagnosis of $\mathrm{CCl} \geq 42.1$.

Concerning the previously published studies exploring the role of the CONUT score in LT, our study presents some beneficial aspects.

As an example, our analysis was performed on HCC patients and patients with an acute or a severe chronic endstage liver disease (ESLD). As well known, ESLD causes a reduction in the biosynthetic activity of the liver, translating into lower levels of circulating proteins such as albumin and apolipoproteins. Consequently, the CONUT score reflects the actual liver functional reserve, being particularly useful in the specific setting of patients with more advanced liver disease (34). As a confirmation of this datum, we observed higher CONUT values and lower median levels of cholesterol and albumin in the high CCI group, namely the group comprising more advanced ESLD cases. When comparing the single components of the CONUT score, albumin showed higher predictive ability compared to cholesterol and lymphocyte count, in regards of post-LT morbidity. Similar findings were obtained in the field of thoracic oncologic surgery, with albumin and CONUT having nearly superimposable AUC values, superior to both cholesterol and lymphocyte count AUCs (35).

Another critical aspect to underline is the statistical approach we adopted with the intent to minimize confounding phenomena. Several potential confounders have been identified to bias our results when we compared the two groups with low or high CCI. For example, patients with a lower CCI were more likely to have HCC and a lower median MELD score (i.e., less severe liver disease). Conversely, patients with a higher CCI presented a longer CIT, potentially caused by the increased complexity and longer duration of the hepatectomy (i.e., more complex surgery due to severe liver disease). Thanks to the use of a stabilized IPTW, we were able to "balance" our population for these potential confounders, therefore eliminating the potential bias caused by their effect.

Interestingly, no statistical difference was detected concerning the donor characteristics even before using the IPTW, further emphasizing the prominent role of the initial ESLD severity in determining post-LT complications. However, no firm assumptions can be drawn in these regards due to the abovementioned limitations.

Another relevant aspect of the present study was that the diagnostic performance of the CONUT score in predicting severe post-LT complications was compared for the first time with other commonly used diagnostic tools for organ allocation and donor-recipient match, namely MELD, MELDNa, and D-MELD score. Interestingly, the CONUT score had the best performance as a pre-operative diagnostic tool for predicting a poor postLT course. The availability of a tool to predict complications is highly desirable and is a topic of central interest in the transplant community, as the complexity of LT procedures continues to increase and more malnourished and immunocompromised patients are evaluated. Similarly to other fields of application, our results confirm the advantages of the CONUT score as a cheap, user-friendly, and pre-operatively available score based on routine blood tests.

Moreover, the pre-transplant CONUT values should consent to target high-risk patients, offering interventions that tackle frailty and sarcopenia before LT (e.g., using nutritional supplementation, immunomodulation, exercise) $(36,37)$.

As an example, a recent study from Italy reported that an "urgency" model combining MELDNa and sarcopenia should be used to prioritize the sarcopenic patients with an initial MELDNa $<20$ on the list, further underlying the relevance of the nutritional status in the LT candidates and the scarce ability of the MELD system in capturing the actual complexity of these patients (38).

The use of rehabilitation programs based on multidisciplinary "training" to enhance physical strength and nutritional status has been proven to increase the physiologic reserve before surgery and withstand complications after transplant (39).

The importance of these considerations is even more critical in light of the evolving epidemiology of LT candidates due to the increased prevalence of non-alcoholic steatohepatitis (NASH). A recent study investigating the relationship between frailty and 


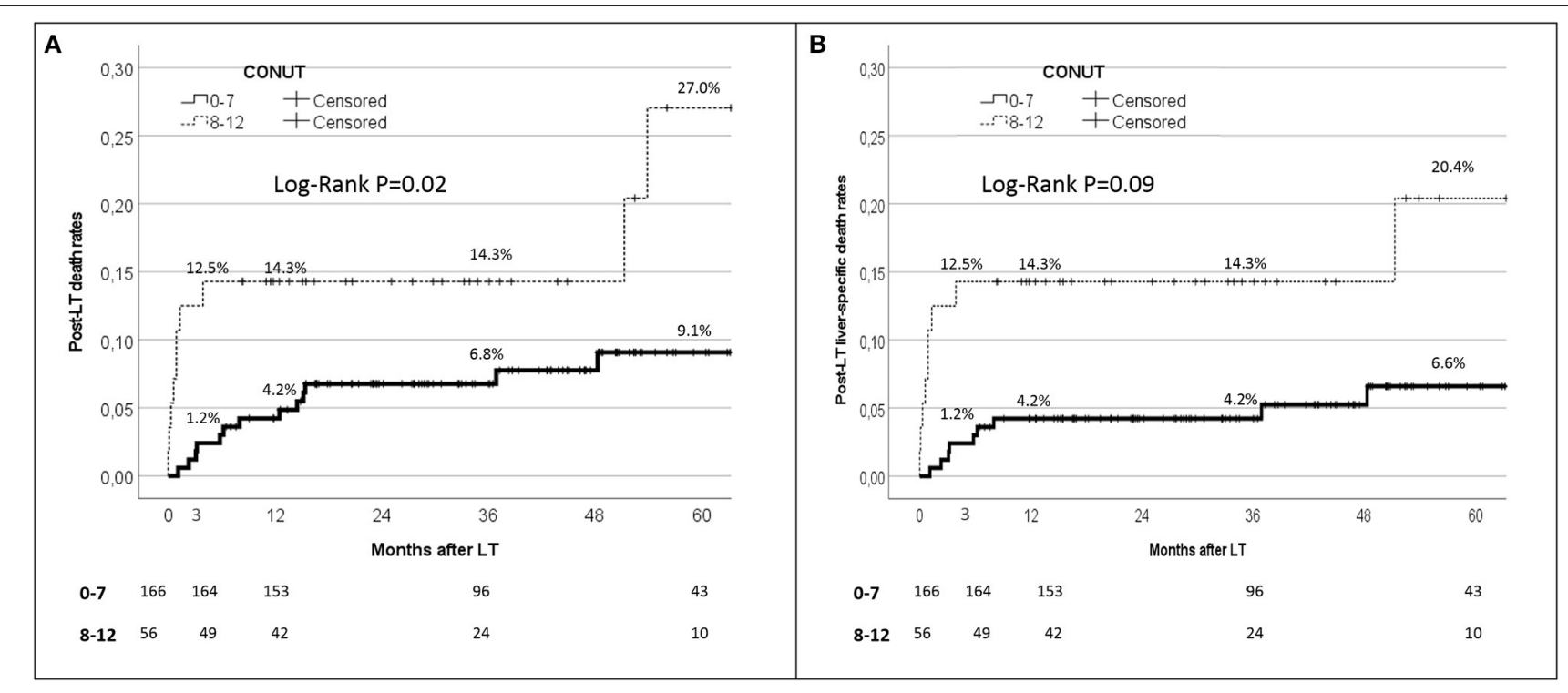

FIGURE 4 | Kaplan-Maier analysis for the 90-day patient survival rates in patient initially with CONUT 0-7 vs 8-12.

cirrhosis etiology revealed that NASH patients were the frailest category of LT candidates, justifying particular attention to the liver functional reserve and malnourishment and immunologic impairment when a patient is transplanted (40).

The CONUT score should play an important role also in the evaluation of the post-LT course, due to the modification of its value in the months after the transplant (33). In this setting, immunosuppression might play a relevant role, mainly impacting on some of the variables composing the CONUT score (e.g., mTOR inhibitors and cholesterol). Further studies are required for the validation of post-transplant CONUT score as a prognosticator of long-term outcomes.

Our study has some limitations. Since this was a retrospective study, the time-point of data collection before LT was heterogeneous. To minimize this heterogeneity, we decided to consider only the blood tests available two months before the transplant. Such a decision impacted the global number of patients we were able to enroll for the study. Many patients transplanted during the study period were not included in the analysis because of outdated tests. The main problem was connected with the cholesterol test, which was not routinely repeated during the LT waitlist. However, despite the consequent sample size reduction, we thought it was a more severe bias to use CONUT calculations based on outdated blood tests (for example, at the time of waiting list inscription), therefore losing the ability of the score to capture the actual nutritional status of the patients at the time of transplantation.

Another already reported limiting factor relates to the number of patients included. Considering our sample size, we were aware that a selection bias could jeopardize the quality of the results of our study. To mitigate such risk, we chose the stabilized IPTW, which allowed us to minimize the effect of potential confounders.
A potential limit to report is that some albumin levels could be partly increased by the intravenous supplementation administered to decompensated ESLD patients. Such practice has been become routine since the supplementation of intravenous human albumin solution was demonstrated to titrate the higher level of prostaglandin PGE2 that is responsible for the macrophage impairment in patients with acutely decompensated cirrhosis (41).

However, only a limited number of patients $(<10 \%)$ in our series present such a condition. Moreover, other commonly used scores carry similar problems (i.e., MELD and plasma infusion). Thus, we considered this limitation unresolvable in the clinical practice and only marginally impacting on the observed results.

Lastly, due to the lack of sufficient data, we could not investigate the importance of decreasing HDL levels in relation to total cholesterol. It has been observed that HDL levels tend to drop proportionally with the evolution of the severity of the ESLD (42). Further studies are needed to investigate if HDL cholesterol levels might further refine the CONUT score in predicting post-LT outcomes.

In conclusion, our study shows a correlation between the CONUT score and the development of severe complications and 90-day as well as long-term mortality after liver transplantation. The CONUT score proved to be a reliable and easy-to-calculate tool that could be integrated in clinical practice with affordable extra costs. Prospective studies are required to corroborate the present findings.

\section{DATA AVAILABILITY STATEMENT}

The raw data supporting the conclusions of this article will be made available by the authors, without undue reservation. 


\section{ETHICS STATEMENT}

Ethical review and approval was not required for the study on human participants in accordance with the local legislation and institutional requirements. Written informed consent for participation was not required for this study in accordance with the national legislation and the institutional requirements.

\section{REFERENCES}

1. Kardashian A, Ge J, McCulloch CE, Kappus MR, Dunn MA, Duarte-Rojo A, et al. Identifying an optimal liver frailty index cutoff to predict waitlist mortality in liver transplant candidates. Hepatol Baltim Md. (2021) 73:11329. doi: 10.1002/hep.31406

2. Guijo-Rubio D, Briceño J, Gutiérrez PA, Ayllón MD, Ciria R, HervásMartínez C. Statistical methods versus machine learning techniques for donor-recipient matching in liver transplantation. PLoS ONE. (2021) 16:e0252068. doi: 10.1371/journal.pone.0252068

3. Skipworth JR, Spoletini G, Imber C. Surgical issues in retrieval and implantation. $\mathrm{Br} J$ Hosp Med Lond Engl. (2017) 78:26672. doi: 10.12968/hmed.2017.78.5.266

4. Agopian VG, Harlander-Locke MP, Markovic D, Dumronggittigule W, Xia V, Kaldas FM, et al. Evaluation of early allograft function using the liver graft assessment following transplantation risk score model. JAMA Surg. (2018) 153:436-44. doi: 10.1001/jamasurg.2017.5040

5. Avolio AW, Franco A, Schlegel A, Lai Q, Meli S, Burra P, et al. Development and validation of a comprehensive model to estimate early allograft failure among patients requiring early liver retransplant. JAMA Surg. (2020) 155:e204095. doi: 10.1001/jamasurg.2020.4095

6. Pravisani R, Mocchegiani F, Isola M, Lorenzin D, Adani GL, Cherchi V, et al. Controlling Nutritional Status score does not predict patients' overall survival or hepatocellular carcinoma recurrence after deceased donor liver transplantation. Clin Transplant. (2020) 34:e13786. doi: 10.1111/ctr.13786

7. Raveh Y, Livingstone J, Mahan J, Tekin A, Selvaggi G, Bowdon-Romero M, et al. Comprehensive frailty severity index for end-stage liver disease predicts early outcomes after liver transplantation. JPEN J Parenter Enteral Nutr. (2020) 44:1079-88. doi: 10.1002/jpen.1729

8. Amygdalos I, Bednarsch J, Meister FA, Erren D, Mantas A, Strnad P, et al. Clinical value and limitations of the pre-operative C-reactive-proteinto-albumin ratio in predicting postoperative morbidity and mortality after deceased-donor liver transplantation: a retrospective single-centre study. Transpl Int Off J Eur Soc Organ Transplant. (2021) 34:146880 doi: $10.1111 /$ tri.13957

9. Jin $\mathrm{H}$, Zhu K, Wang W. The predictive values of pretreatment Controlling Nutritional Status (CONUT) score in estimating short- and long-term outcomes for patients with gastric cancer treated with neoadjuvant chemotherapy and curative gastrectomy. J Gastric Cancer. (2021) 21:15568. doi: 10.5230/jgc.2021.21.e14

10. Wang A, He Z, Cong P, Qu Y, Hu T, Cai Y, et al. Controlling Nutritional Status (CONUT) score as a new indicator of prognosis in patients with hilar cholangiocarcinoma is superior to NLR and PNI: a single-center retrospective study. Front Oncol. (2020) 10:593452. doi: 10.3389/fonc.2020.593452

11. Müller L, Hahn F, Mähringer-Kunz A, Stoehr F, Gairing SJ, Foerster F, et al. Immunonutritive scoring in patients with hepatocellular carcinoma undergoing transarterial chemoembolization: prognostic nutritional index or controlling nutritional status score? Front Oncol. (2021) 11:696183. doi: $10.3389 /$ fonc. 2021.696183

12. Ignacio de. Ulíbarri J, González-Madroño A, de Villar NGP, González P, González B, Mancha A, et al. CONUT: a tool for controlling nutritional status First validation in a hospital population. Nutr Hosp. (2005) 20:38-45.

13. Yagi T, Oshita Y, Okano I, Kuroda T, Ishikawa K, Nagai T, et al. Controlling nutritional status score predicts postoperative complications after hip fracture surgery. BMC Geriatr. (2020) 20:243. doi: 10.1186/s12877-020-01643-3

14. Shiihara M, Higuchi R, Izumo W, Yazawa T, Uemura S, Furukawa T, et al. Impact of the controlling nutritional status score on severe postoperative

\section{AUTHOR CONTRIBUTIONS}

GS and QL: conception and design. SA and MR: administrative support. SA, MR, and AWA: provision of study materials or patients. FF, GB, AM, and QL: collection and assembly of data. QL, AWA, and GS: data analysis and interpretation. All authors: manuscript writing and final approval of manuscript.

complications of pancreaticoduodenectomy for pancreatic cancer. Langenbecks Arch Surg. (2021) 406:1491-8. doi: 10.1007/s00423-021-02151-7

15. Dong X, Tang S, Liu W, Qi W, Ye L, Yang X, et al. Prognostic significance of the Controlling Nutritional Status (CONUT) score in predicting postoperative complications in patients with Crohn's disease. Sci Rep. (2020) 10:19040. doi: 10.1038/s41598-020-76115-0

16. Yoshida N, Baba Y, Shigaki H, Harada K, Iwatsuki M, Kurashige J, et al. Preoperative nutritional assessment by Controlling Nutritional Status (CONUT) is useful to estimate postoperative morbidity after esophagectomy for esophageal cancer. World J Surg. (2016) 40:19107. doi: 10.1007/s00268-016-3549-3

17. Clavien PA, Barkun J, de Oliveira ML, Vauthey JN, Dindo D, Schulick RD, et al. The Clavien-Dindo classification of surgical complications: five-year experience. Ann Surg. (2009) 250:187-96. doi: 10.1097/SLA.0b013e3181b13ca2

18. Slankamenac K, Graf R, Barkun J, Puhan MA, Clavien PA. The comprehensive complication index: a novel continuous scale to measure surgical morbidity. Ann Surg. (2013) 258:17. doi: 10.1097/SLA.0b013e318296c732

19. Lai Q, Melandro F, Nowak G, Nicolini D, Iesari S, Fasolo E, et al. The role of the comprehensive complication index for the prediction of survival after liver transplantation. Updat Surg. (2021) 73:209-21. doi: 10.1007/s13304-020-00878-4

20. Muller X, Marcon F, Sapisochin G, Marquez M, Dondero F, Rayar M, et al. Defining benchmarks in liver transplantation: a multicenter outcome analysis determining best achievable results. Ann Surg. (2018) 267:41925. doi: 10.1097/SLA.0000000000002477

21. Zhang Z. Missing data imputation: focusing on single imputation. Ann Transl Med. (2016) 4:9. doi: 10.3978/j.issn.2305-5839.2015.12.38

22. Burnand B, Kernan WN, Feinstein AR. Indexes and boundaries for "quantitative significance" in statistical decisions. J Clin Epidemiol. (1990) 43:1273-84. doi: 10.1016/0895-4356(90)90093-5

23. Fozouni L, Mohamad Y, Lebsack A, Freise C, Stock P, Lai JC. Frailty is associated with increased rates of acute cellular rejection within 3 months after liver transplantation. Liver Transplant Off Publ Am Assoc Study Liver Dis Int Liver Transplant Soc. (2020) 26:390-6. doi: 10.1002/lt.25669

24. Burra P, Samuel D, Sundaram V, Duvoux C, Petrowsky H, Terrault N, et al. Limitations of current liver donor allocation systems and the impact of newer indications for liver transplantation. J Hepatol. (2021) 75 Suppl 1:S178-90. doi: 10.1016/j.jhep.2021.01.007

25. Li L, Liu C, Yang J, Wu H, Wen T, Wang W, et al. Early postoperative controlling nutritional status (CONUT) score is associated with complication III-V after hepatectomy in hepatocellular carcinoma: a retrospective cohort study of 1,334 patients. Sci Rep. (2018) 8:13406. doi: 10.1038/s41598-018-31714-w

26. Miyata T, Yamashita Y-I, Higashi T, Taki K, Izumi D, Kosumi K, et al. The prognostic impact of Controlling Nutritional Status (CONUT) in intrahepatic cholangiocarcinoma following curative hepatectomy: a retrospective single institution study. World J Surg. (2018) 42:108591. doi: 10.1007/s00268-017-4214-1

27. Harimoto $\mathrm{N}$, Yoshizumi $\mathrm{T}$, Inokuchi $\mathrm{S}$, Itoh $\mathrm{S}$, Adachi $\mathrm{E}$, Ikeda $\mathrm{Y}$, et al. Prognostic significance of preoperative Controlling Nutritional Status (CONUT) score in patients undergoing hepatic resection for hepatocellular carcinoma: a multi-institutional study. Ann Surg Oncol. (2018) 25:331623. doi: 10.1245/s10434-018-6672-6

28. Iseki Y, Shibutani M, Maeda K, Nagahara H, Ohtani H, Sugano K, et al. Impact of the preoperative Controlling Nutritional Status (CONUT) score 
on the survival after curative surgery for colorectal cancer. PLoS ONE. (2015) 10:e0132488. doi: 10.1371/journal.pone.0132488

29. Toyokawa T, Kubo N, Tamura T, Sakurai K, Amano R, Tanaka H, et al. The pretreatment Controlling Nutritional Status (CONUT) score is an independent prognostic factor in patients with resectable thoracic esophageal squamous cell carcinoma: results from a retrospective study. BMC Cancer. (2016) 16:722. doi: 10.1186/s12885-016-2696-0

30. Toyokawa G, Kozuma Y, Matsubara T, Haratake N, Takamori S, Akamine T, et al. Prognostic impact of controlling nutritional status score in resected lung squamous cell carcinoma. J Thorac Dis. (2017) 9:294251. doi: 10.21037/jtd.2017.07.108

31. Kuroda D, Sawayama H, Kurashige J, Iwatsuki M, Eto T, Tokunaga $\mathrm{R}$, et al. Controlling Nutritional Status (CONUT) score is a prognostic marker for gastric cancer patients after curative resection. Gastric Cancer Off J Int Gastric Cancer Assoc Jpn Gastric Cancer Assoc. (2018) 21:20412. doi: 10.1007/s10120-017-0744-3

32. Ishihara H, Kondo T, Yoshida K, Omae K, Takagi T, Iizuka J, et al. Preoperative controlling nutritional status (CONUT) score as a novel predictive biomarker of survival in patients with localized urothelial carcinoma of the upper urinary tract treated with radical nephroureterectomy. Urol Oncol. (2017) 35:539.e9-16. doi: 10.1016/j.urolonc.2017.04.012

33. Pravisani R, Mocchegiani F, Isola M, Lorenzin D, Adani GL, Cherchi V, et al. Postoperative trends and prognostic values of inflammatory and nutritional biomarkers after liver transplantation for hepatocellular carcinoma. Cancers. (2021) 13:513. doi: 10.3390/cancers13030513

34. Nishikawa H, Yoh K, Enomoto H, Ishii N, Iwata Y, Takata R, et al. The Relationship between Controlling Nutritional (CONUT) score and clinical markers among adults with hepatitis $\mathrm{C}$ virus related liver cirrhosis. Nutrients. (2018) 10:1185. doi: 10.3390/nu10091185

35. Takamori S, Toyokawa G, Taguchi K, Edagawa M, Shimamatsu S, Toyozawa $\mathrm{R}$, et al. The controlling nutritional status score is a significant independent predictor of poor prognosis in patients with malignant pleural mesothelioma. Clin Lung Cancer. (2017) 18:e303-13. doi: 10.1016/j.cllc.2017.01.008

36. Lai JC, Covinsky KE, Dodge JL, Boscardin WJ, Segev DL, Roberts JP, et al. Development of a novel frailty index to predict mortality in patients with end-stage liver disease. Hepatol Baltim Md. (2017) 66:56474. doi: 10.1002/hep.29219

37. Duarte-Rojo A, Ruiz-Margáin A, Montaño-Loza AJ, Macías-Rodríguez RU, Ferrando A, Kim WR. Exercise and physical activity for patients with end-stage liver disease: Improving functional status and sarcopenia while on the transplant waiting list. Liver Transplant. (2018) 24:12239. doi: 10.1002/lt.24958

38. Lai Q, Magistri P, Lionetti R, Avolio AW, Lenci I, Giannelli V, et al. Sarcomodel: a score to predict the dropout risk in the perspective of organ allocation in patients awaiting liver transplantation. Liver Int. (2021) 41:162940. doi: 10.1111/liv.14889

39. Lin F-P, Visina JM, Bloomer PM, Dunn MA, Josbeno DA, Zhang X, et al. Prehabilitation-driven changes in frailty metrics predict mortality in patients with advanced liver disease. Am J Gastroenterol. (2021) 116:210517 doi: 10.14309/ajg.0000000000001376

40. Xu CQ, Mohamad Y, Kappus MR, Boyarsky B, Ganger DR, Volk ML, et al. The relationship between frailty and cirrhosis etiology: from the Functional Assessment in Liver Transplantation (FrAILT) Study. Liver Int Off J Int Assoc Study Liver. (2021) 41:2467-73 doi: 10.1111/liv.15006

41. O'Brien AJ, Fullerton JN, Massey KA, Auld G, Sewell G, James S, et al. Immunosuppression in acutely decompensated cirrhosis is mediated by prostaglandin E2. Nat Med. (2014) 20:518-23. doi: 10.1038/ nm.3516

42. Katsiki N, Mikhailidis DP, Mantzoros CS. Non-alcoholic fatty liver disease and dyslipidemia: an update. Metabolism. (2016) 65:1109-23. doi: 10.1016/j.metabol.2016.05.003

Conflict of Interest: The authors declare that the research was conducted in the absence of any commercial or financial relationships that could be construed as a potential conflict of interest.

Publisher's Note: All claims expressed in this article are solely those of the authors and do not necessarily represent those of their affiliated organizations, or those of the publisher, the editors and the reviewers. Any product that may be evaluated in this article, or claim that may be made by its manufacturer, is not guaranteed or endorsed by the publisher.

Copyright (c) 2022 Spoletini, Ferri, Mauro, Mennini, Bianco, Cardinale, Agnes, Rossi, Avolio and Lai. This is an open-access article distributed under the terms of the Creative Commons Attribution License (CC BY). The use, distribution or reproduction in other forums is permitted, provided the original author(s) and the copyright owner(s) are credited and that the original publication in this journal is cited, in accordance with accepted academic practice. No use, distribution or reproduction is permitted which does not comply with these terms. 